\title{
Article \\ A Real-World Comparative Study of Microwave and Radiofrequency Ablation in Treatment-Naïve and Recurrent Hepatocellular Carcinoma
}

\author{
Soon Kyu Lee ${ }^{1}$, Dong Jin Chung ${ }^{2}$ and Se Hyun Cho ${ }^{1, *}$ \\ 1 Division of Gastroenterology and Hepatology, Department of Internal Medicine, College of Medicine, \\ The Catholic University of Korea, Seoul 06591, Korea; blackiqq@catholic.ac.kr \\ 2 Department of Radiology, College of Medicine, The Catholic University of Korea, Seoul 06591, Korea; \\ bookdoo7@catholic.ac.kr \\ * Correspondence: chowhang@catholic.ac.kr; Tel.: +82-2-3779-2330
}

Citation: Lee, S.K.; Chung, D.J.; Cho, S.H. A Real-World Comparative Study of Microwave and

Radiofrequency Ablation in

Treatment-Naïve and Recurrent

Hepatocellular Carcinoma. J. Clin.

Med. 2022, 11, 302. https://doi.org/

$10.3390 /$ jcm11020302

Academic Editor: Hiroyuki Isayama

Received: 16 October 2021

Accepted: 6 January 2022

Published: 7 January 2022

Publisher's Note: MDPI stays neutral with regard to jurisdictional claims in published maps and institutional affiliations.

Copyright: (C) 2022 by the authors. Licensee MDPI, Basel, Switzerland. This article is an open access article distributed under the terms and conditions of the Creative Commons Attribution (CC BY) license (https:// creativecommons.org/licenses/by/ $4.0 /)$.

\begin{abstract}
The efficacy and safety of microwave ablation (MWA) compared to radiofrequency ablation (RFA) for patients with treatment-naïve and recurrent hepatocellular carcinoma (HCC) has not been clarified in Korea. There were 150 HCC patients (100 in the RFA group and 50 in the MWA group) enrolled in our study. The primary outcome was one- and two-year disease-free survival (DFS). Secondary outcomes were complete response (CR) rate, two-year survival rate, risk factors for DFS and complication rate. Treatment outcomes were also assessed using propensity-score matching (PSM). The MWA group had better one- and two-year DFS than the RFA group $(p=0.035$ and $p=0.032$, respectively), whereas the CR rate, two-year survival rate, and complication rate were similar between the two groups with fewer major complications in the MWA group $(p=0.043)$. Patients with perivascular tumors, high risk of recurrence, and small tumor size $(\leq 3 \mathrm{~cm})$ were more suitable for MWA than RFA. MWA was also an independent factor for favorable one- and two-year DFS. Finally, the MWA group still showed better one- and two-year DFS than the RFA group after PSM. In conclusion, MWA could be an alternative treatment to RFA especially in patients with a high risk of recurrence, perivascular tumors, and small tumor size.
\end{abstract}

Keywords: hepatocellular carcinoma; ablation; microwave; radiofrequency; disease-free survival

\section{Introduction}

Hepatocellular carcinoma (HCC) is the fifth most common cancer and the second leading cause of cancer-associated mortality [1,2]. The poor prognosis of HCC is mainly due to late diagnosis at an advanced stage, which prohibits the application of curative therapies [3]. To improve the prognosis of HCC by diagnosing HCC at an early stage, a surveillance strategy using alpha-fetoprotein (AFP) and ultrasonography every six months is recommended for at-risk populations, including those with liver cirrhosis (LC) [4,5].

Early detection of small HCC provides the chance for curative treatments such as liver transplantation, liver resection (LR), and radiofrequency ablation (RFA) [6]. However, patients with HCC are still exposed to the risk of recurrence after curative treatments. Indeed, these patients usually have chronic liver diseases including LC, and HCC can recur not only within five years but also more than five years after curative treatments [7]. Therefore, early diagnosis of HCC as well as curative treatments with a low rate of recurrence are the cornerstones for improving the prognosis of patients with HCC.

RFA is the standard of care for early HCC and is comparable with LR in solitary HCCs smaller than $3 \mathrm{~cm}$ [8]. RFA treats tumor cells by the generation of an electric current, which causes local heat with a temperature of $60-100{ }^{\circ} \mathrm{C}$ that can lead to thermocoagulation necrosis [9]. However, as RFA heats the target area via thermal conduction, the active heating zone around electrodes is only a few millimeters, which causes a decrease in the 
treatment efficacy for tumors larger than $2-3 \mathrm{~cm}$ or those located near a major vessel $[10,11]$. To complement these limitations, new ablation methods have been developed including microwave ablation (MWA).

MWA, which generates an electromagnetic field, can theoretically reach a higher temperature in a shorter time, create a larger ablation zone, and is less affected by adjacent tissues than RFA [12]. Based on these advantages, MWA has been compared to RFA in several studies, and seems to be an alternative treatment to RFA [13]. However, there has been no real-life comparative study of MWA and RFA in Korea. Moreover, the treatment outcomes of MWA, including patients with recurrent HCC, have not been elucidated.

Herein, we evaluated the treatment outcomes of MWA compared to RFA in Korea for the first time. To reflect real-world data, we also included patients with recurrent HCC and employed propensity-score matching (PSM) analysis. In addition, risk factors for disease-free survival (DFS) and subgroups favoring MWA were also evaluated.

\section{Materials and Methods}

\subsection{Study Population}

In our retrospective cohort study, a total of $261 \mathrm{HCC}$ patients treated with ablation therapy (RFA or MWA) from September 2014 to December 2020 at Yeouido St. Mary's Hospital (Seoul, Korea) were consecutively enrolled. Of these patients, 111 patients were excluded for the following reasons: ablation therapy for metastatic lesions $(n=77)$, history of both procedures $(n=26)$, intraoperative procedure $(n=2)$, and follow-up of less than three months $(n=6)$. Finally, 150 patients with HCC who were treated with RFA $(n=100)$ or MWA $(n=50)$ were included and analyzed (Supplementary Figure S1). This study was approved by the Institutional Review Board of Yeouido St. Mary's Hospital (SC21RISI0023), and the requirement to obtain informed consent was waived. The study was conducted in accordance with the Declaration of Helsinki.

\subsection{Ablation Therapies}

All treatment procedures (RFA and MWA) were performed by an experienced radiologist under monitored anesthesia care. Ultrasound-guided percutaneous ablation was performed during the procedure to monitor treatment. RFA was performed using an RFA system (M-3004; 200-W multifunctional generator; RF Medical, Seoul, Korea) with a 15-G needle electrode. For MWA, an Emprint ablation system (Medtronic, Dublin, UK) with a power of up to $100 \mathrm{~W}$ was used. A 13-G straight internally cooled antenna with a frequency of $2450 \mathrm{MHz}$ was applied for MWA therapy. After ablation therapy, patients were monitored for one or two days to observe complications.

\subsection{Diagnosis and Follow-Up}

HCC was diagnosed either by histology or according to the guidelines of the European Association for the Study of the Liver or the American Association for the Study of Liver Disease $[4,14,15]$. Perivascular HCC was defined based on previous studies using multiphase contrast-enhanced computed tomography (CT) or magnetic resonance imaging (MRI) [16,17]. The tumor stage was classified according to the modified Union for International Cancer Control (mUICC) stage and Barcelona Clinic Liver Cancer (BCLC) stage $[15,18]$.

Technical success was evaluated immediately after ablation therapy by $\mathrm{CT}$, and achievement of complete response (CR) was assessed by CT or MRI two or three months after ablation therapy. Follow-up CT or MRI was performed every three months to evaluate tumor response according to the modified Response Evaluation Criteria in Solid Tumors (mRECIST) [19]. Patients with recurrence after RFA or MWA were treated appropriately according to HCC treatment guidelines [14,15,18]. 


\subsection{Definitions and Outcome Measures}

Since MWA has been used in recent years of the study, the primary endpoint was one- and two-year DFS, defined as the time interval between the time of RFA or MWA and the time of recurrence, death, or the last follow-up in patients without recurrence, respectively. The second outcomes of the study were two-year overall survival (OS), CR rate, local recurrence rate, and complications. OS was defined as the time interval between the procedure (RFA or MWA) and death or the last follow-up time. The definition of local recurrence was recurrence of the tumor at the adjacent site of treated lesion $(\leq 2.0 \mathrm{~cm}$ from its margin) [20]. Patients were monitored for complications after treatment and classified following the definitions of the Society of Interventional Radiology [21]. Major complications included events requiring therapies, prolonged hospitalization, morbidity, or death, with other complications considered as minor.

\subsection{Statistical Analysis}

Baseline characteristics of patients were expressed as means \pm standard deviation for quantitative variables, and as counts (percentage) for categorical variables, as appropriate. Between-group comparisons including the rate of $\mathrm{CR}$, local recurrence, and complications were analyzed by Student's $t$-test, the chi-squared test, or Fisher's exact test, as appropriate. Kaplan-Meier analyses were used to demonstrate one- and two-year DFS, and two-year OS according to treatment groups (MWA and RFA group) and subgroups. Since MWA has been used in recent years of the study, we performed log-rank tests truncated at 12 or 24 months of follow-up, as appropriate. To evaluate the risk factors for one- and two-year DFS and two-year OS, significant variables $(p<0.05)$ in univariate analysis were introduced into the multivariate Cox regression method with a backward conditional method.

Moreover, to reduce selection bias, a one-to-one nearest-neighbor PSM analysis was performed by equating the RFA and MWA groups based on the following variables: sex, age, prior treatment history, cause of HCC, presence of cirrhosis, Child-Turcotte-Pugh (CTP) class, Model for End-stage Liver Disease (MELD) score, tumor size, tumor number, perivascular tumor, BCLC stage, and mUICC stage. $p$-values $<0.05$ were considered significant and all statistical analyses were performed using SPSS version 24.0 (IBM, Armonk, NY, USA) and R version 4.0.4 (R Foundation for Statistical Computing, Vienna, Austria).

\section{Results}

\subsection{Baseline Characteristics}

The mean age of participants was 66.0 years and most patients $(92.7 \%)$ had CTP class A. Of the 150 included patients, $108(72.0 \%)$ were male and $122(82.0 \%)$ had LC. Proportions of patients with a single tumor $(73.0 \%$ vs. $88.0 \%, p=0.060)$ and perivascular tumors $(21.0 \%$ vs. $34.0 \%, p=0.127)$ were not significantly different between the RFA and MWA groups. However, the MWA group $(n=50)$ had more treatment-naïve patients $(50 \%$ vs. $28 \%, p=0.013)$ and larger tumor size $(2.1$ vs. $1.9 \mathrm{~cm}, p=0.046)$ than the RFA group (Table 1). 
Table 1. Baseline characteristics of the entire population (general and propensity-score matching model).

\begin{tabular}{|c|c|c|c|c|c|c|c|}
\hline \multirow{2}{*}{ Variables } & \multirow{2}{*}{$\begin{array}{c}\text { Total } \\
(n=150)\end{array}$} & \multicolumn{3}{|c|}{ General Model } & \multicolumn{3}{|c|}{ Propensity-Score Matching Model } \\
\hline & & RFA Group $(n=100)$ & MWA Group $(n=50)$ & $p$-Value & RFA Group $(n=42)$ & MWA Group $(n=42)$ & $p$-Value \\
\hline Male sex $(n, \%)$ & $108(72.0 \%)$ & $71(71.0 \%)$ & $37(74.0 \%)$ & 0.847 & $30(71.4 \%)$ & $32(76.2 \%)$ & 0.804 \\
\hline Age, years & $66.0 \pm 10.2$ & $65.2 \pm 9.9$ & $67.7 \pm 10.5$ & 0.152 & $68.4 \pm 8.9$ & $66.2 \pm 10.4$ & 0.314 \\
\hline Treatment naïve $(n, \%)$ & $53(35.3 \%)$ & $28(28.0 \%)$ & $25(50.0 \%)$ & 0.013 & $18(42.9 \%)$ & $20(47.6 \%)$ & 0.826 \\
\hline Cause $(n, \%)$ & & & & 0.822 & & & 0.781 \\
\hline Viral/Non-viral & $123(82.0 \%) / 27(18.0 \%)$ & $83(83.0 \%) / 17(17.0 \%)$ & $40(80.0 \%) / 10(20.0 \%)$ & & $35(83.3 \%) / 7(16.7 \%)$ & $33(78.6 \%) / 9(21.4 \%)$ & \\
\hline Cirrhosis & $122(81.3 \%)$ & $83(83.0 \%)$ & $39(78.0 \%)$ & 0.604 & $31(73.8 \%)$ & $34(81.0 \%)$ & 0.602 \\
\hline CTP class $(n, \%)$ & & & & 1.000 & & & 1.000 \\
\hline Class A/class B & $139(92.7 \%) / 11(7.3 \%)$ & $93(93.0 \%) / 7(7.0 \%)$ & $46(92.0 \%) / 4(8.0 \%)$ & & $41(97.6 \%) / 1(2.4 \%)$ & $40(95.2 \%) / 2(4.8 \%)$ & \\
\hline $\mathrm{AFP}(\mathrm{ng} / \mathrm{mL})$ & $63.4 \pm 188.7$ & $57.2 \pm 161.7$ & $75.9 \pm 234.9$ & 0.614 & $67.6 \pm 198.7$ & $66.1 \pm 240.3$ & 0.975 \\
\hline MELD score & $5.8 \pm 2.6$ & $6.0 \pm 3.0$ & $5.4 \pm 1.7$ & 0.077 & $5.3 \pm 1.2$ & $5.5 \pm 1.6$ & 0.516 \\
\hline Tumor size $(\mathrm{cm})$ & $2.0 \pm 0.8$ & $1.9 \pm 0.7$ & $2.1 \pm 0.9$ & 0.046 & $2.0 \pm 0.9$ & $2.1 \pm 0.8$ & 0.909 \\
\hline Tumor number $(n, \%)$ & & & & 0.060 & & & 1.000 \\
\hline Single & $117(78.0 \%)$ & $73(73.0 \%)$ & $44(88.0 \%)$ & & $37(88.1 \%)$ & $37(88.1 \%)$ & \\
\hline Multiple (2-3) & $33(22.0 \%)$ & $27(27.0 \%)$ & $6(12.0 \%)$ & & $5(11.9 \%)$ & $5(11.9 \%)$ & \\
\hline Perivascular tumor $(n, \%)$ & $38(25.3 \%)$ & $21(21.0 \%)$ & $17(34.0 \%)$ & 0.127 & $12(28.6 \%)$ & $12(28.6 \%)$ & 1.000 \\
\hline BCLC stage $(n, \%)$ & & & & 0.326 & & & 0.662 \\
\hline Stage 0 & $76(50.7 \%)$ & $54(54.0 \%)$ & $22(44.0 \%)$ & & $22(52.4 \%)$ & $19(45.2 \%)$ & \\
\hline Stage A, B & $74(49.3 \%)$ & $46(46.0 \%)$ & $28(56.0 \%)$ & & $20(47.6 \%)$ & $23(54.8 \%)$ & \\
\hline mUICC stage $(n, \%)$ & & & & 0.326 & & & 0.662 \\
\hline Stage I & 76 (50.7\%) & $54(54.0 \%)$ & $22(44.0 \%)$ & & $22(52.4 \%)$ & $19(45.2 \%)$ & \\
\hline Stage II, III & $74(47.3 \%)$ & $46(46.0 \%)$ & $28(56.0 \%)$ & & $20(47.6 \%)$ & $23(54.8 \%)$ & \\
\hline
\end{tabular}


About half of the patients ( $54.0 \%$ vs. $44.0 \%$ ) had BCLC stage 0 and the other patients $(46.0 \%$ vs. $56.0 \%)$ had stage A or B without significant differences between the RFA and MWA groups $(p=0.326)$. Most of recurrent HCC patients included in the study had a treatment history of trans-arterial chemoembolization without significant group differences (MWA, $80 \%$ vs. RFA, $87.5 \% ; p=0.358$ ). The median follow-up duration of the entire population, the MWA, and the RFA group were 25.3 (Interquartile range [IQR], 12.5-46.1 months), 12.2 (IQR, 9.0-16.1 months), and 39.5 (IQR, 24.9-53.4 months), respectively.

\subsection{Treatment Outcomes in the Entire Population}

All patients achieved treatment success after MWA or RFA treatment. During the two-year follow-up, eight patients died due to HCC progression $(n=7)$ or hepatic failure $(n=1)$. OS at two years was similar $(91.7 \%$ vs. $92.4 \%, p=0.573)$ between the MWA and RFA groups (Figure 1A). However, the MWA group had significantly better one-year DFS than the RFA group (79.7\% vs. $60.7 \%, p=0.035$, respectively) (Figures $1 \mathrm{~B}$ and $2 \mathrm{~A}$ ). DFS at two years was also better in the MWA group than in the RFA group $(72.5 \%$ vs. $45.4 \%$, $p=0.020$, respectively) (Figures $1 \mathrm{C}$ and $2 \mathrm{~B}$ ).

(A)

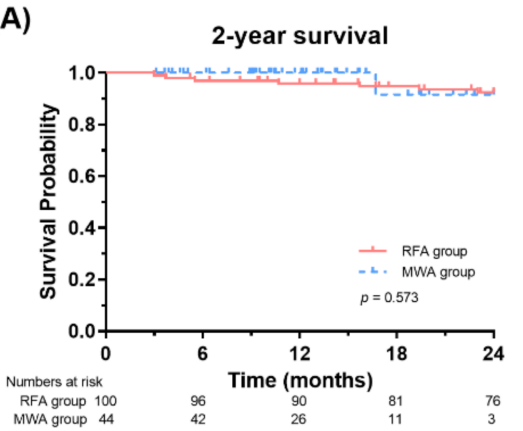

(B)

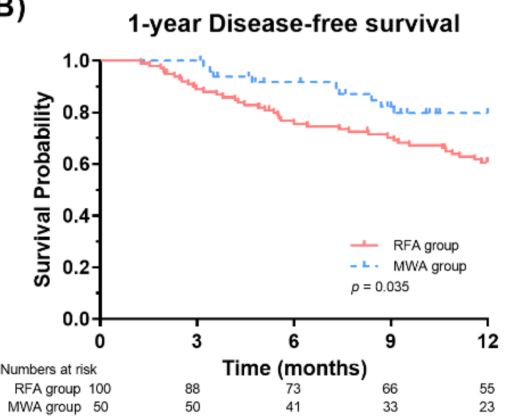

(C)

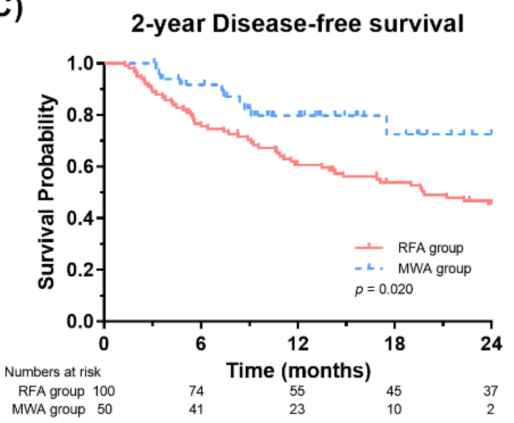

Figure 1. Survival and disease-free survival curves according to the treatment modalities in the entire cohort (A-C). RFA, radiofrequency ablation; MWA, microwave ablation.

(A)

Recurrence within 1 year

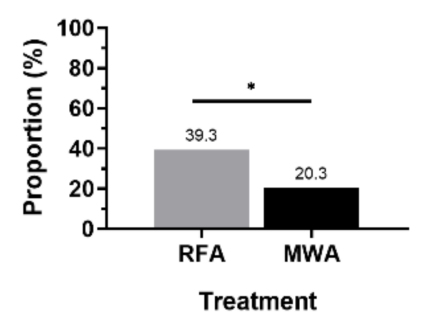

(B)

Recurrence within 2 years

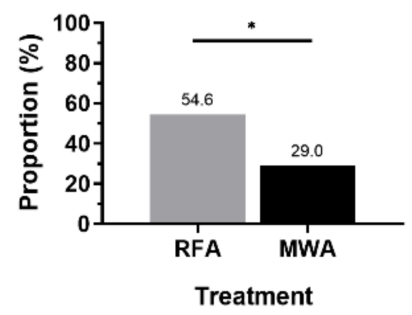

(C)

\section{Complete response rate}

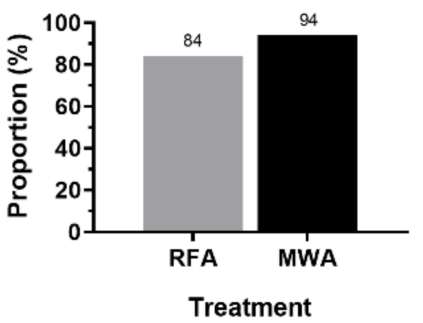

Figure 2. Comparison of recurrent rate (A,B) and treatment response $(\mathbf{C})$ between RFA and MWA. RFA, radiofrequency ablation; MWA, microwave ablation $\left({ }^{*} p<0.05\right)$.

The CR rate was marginally higher in the MWA group than in the RFA group $(94.0 \%$ vs. $84.0 \%, p=0.083$, respectively) (Figure 2C). Among patients with recurrence, we analyzed the differences in the site of recurrence between the two groups and the proportion of local recurrence were similar between the two groups at one year $(p=0.466)$ and two years $(p=0.289)$ (Supplementary Figure S2). During the two-year follow-up, trans-arterial chemoembolization was the most frequent treatment method $(56.9 \%$ vs. $80 \%$, respectively) after recurrence, followed by RFA or MWA (39.2\% vs. $20.0 \%$, respectively) without significant difference between RFA and MWA groups (Supplementary Table S1). 


\subsection{Subgroup Analysis}

We further evaluated the respective outcomes in patients with a high risk of recurrence, including LC and increased AFP levels. In patients with LC and increased AFP levels, the MWA group had a better two-year DFS ( $p=0.035$ and $p=0.032$, respectively) than the RFA group (Figure $3 \mathrm{~A}, \mathrm{~B})$. When analyzed by tumor site and size, the MWA group achieved significantly better two-year DFS in subgroups with perivascular tumors ( $p=0.045$, Figure 3C) and tumor size $\leq 3 \mathrm{~cm}(p=0.046$, Figure 3D) than the RFA group. In the analyses according to the BCLC stage, the MWA group exhibited better two-year DFS than the RFA group in patients with BCLC stage 0 ( $p=0.016$, Figure $3 \mathrm{E})$, whereas patients with BCLC stage A or B showed marginally better two-year DFS in the MWA group than in the RFA group $(p=0.179$, Figure $3 F)$.

(A)

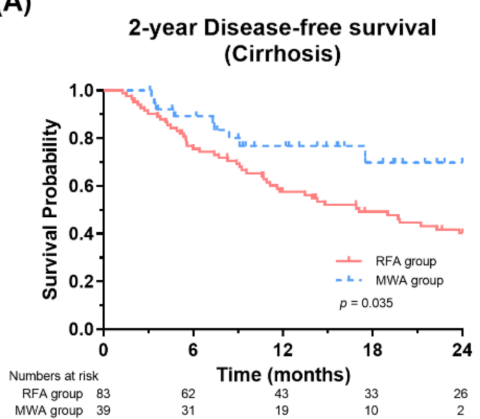

(D)

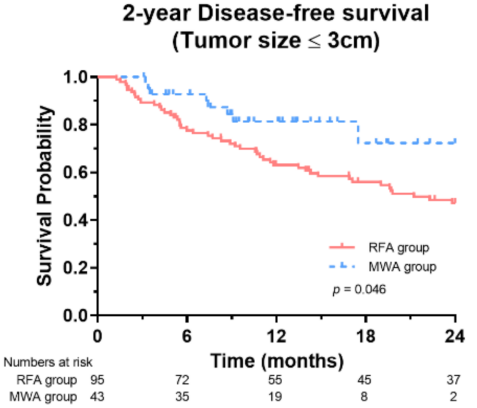

(B)

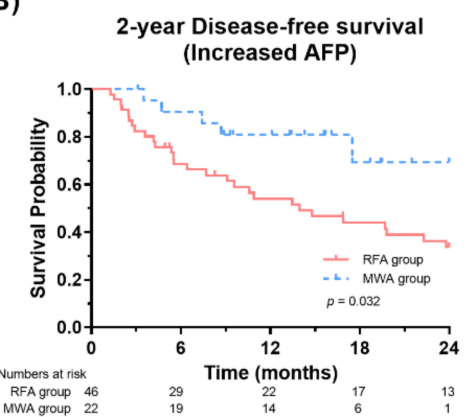

(E)

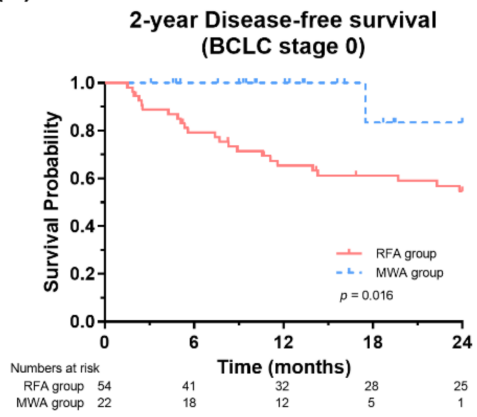

(C)

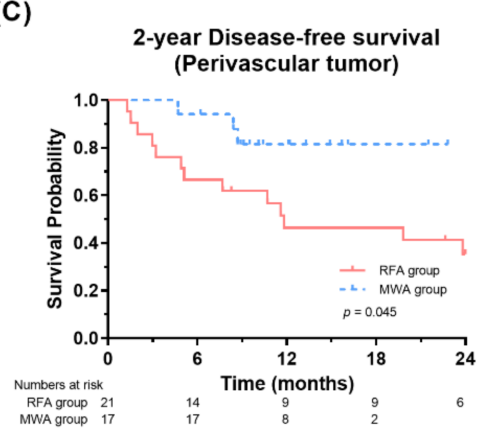

(F)

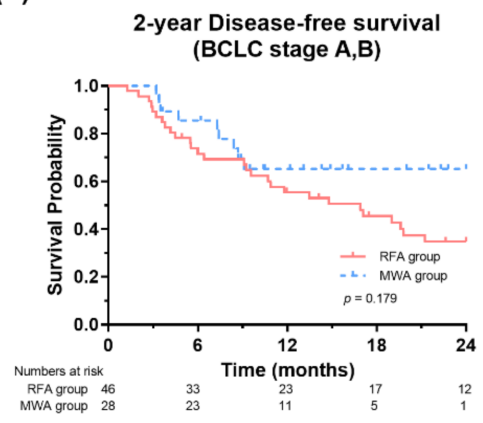

Figure 3. Comparison of disease-free survival in the MWA and RFA groups in subgroups with (A) cirrhosis, (B) increased AFP, (C) perivascular tumor, (D) small tumor size $(\leq 3 \mathrm{~cm})$, and (E,F) BCLC stage 0 , A and B. RFA, radiofrequency ablation; MWA, microwave ablation; AFP, alpha-fetoprotein; BCLC, Barcelona Clinic Liver.

\subsection{Treatment Complications in the Entire Population}

Table 2 shows the summarized data on complications in both groups. Of the 150 included patients, $48(48.0 \%)$ in the RFA group and $19(38.0 \%)$ in the MWA group experienced complications $(p=0.246)$. The MWA group showed a lower rate of major complications than the RFA group (7 of 50 patients [14.0\%] vs. 29 of 100 patients [29.0\%], $p=0.043$, respectively) and pain requiring treatment was the most common major complication in both groups (MWA, $n=7$; RFA, $n=27$ ). Fever and pain were the most common minor complications in both groups without significant group differences $(p=0.476)$. All patients who experienced complications $(n=67)$ after ablation therapy were well recovered and tolerable after appropriate management. 
Table 2. Comparison of treatment complications between RFA and MWA in the entire population.

\begin{tabular}{cccc}
\hline Complications & RFA Group $(\boldsymbol{n}=\mathbf{1 0 0})$ & MWA Group $(\boldsymbol{n}=\mathbf{5 0 )}$ & $\boldsymbol{p}$-Value \\
\hline All complications & $48(48.0 \%)$ & $19(38.0 \%)$ & 0.246 \\
Major complications & $29(29.0 \%)$ & $7(14.0 \%)$ & 0.043 \\
pain requiring treatment & 27 & 7 & \\
Hydrothorax with drain & 1 & 0 & \\
Hemoperitoneum & 1 & 0 & \\
Minor complications & $19(19.0 \%)$ & $12(24.0 \%)$ & \\
pain without treatment & 10 & 8 & \\
Fever without infection & 6 & 3 & \\
Nausea and vomiting & 3 & 1 & \\
\hline
\end{tabular}

RFA, radiofrequency ablation; MWA, microwave ablation.

\subsection{Factors Associated with DFS}

Of the variables listed in Table 3, treatment-naïve tumors, treatment modality, AFP level, and tumor size were associated with one-year DFS, whereas tumor number, perivascular tumor, and BCLC stage were not significantly associated with one-year DFS. In the multivariate analysis, treatment-naïve tumors (hazard ratio [HR], $0.41 ; 95 \%$ confidence interval [CI], 0.20-0.85; $p=0.017$ ), MWA (HR, $0.42 ; 95 \%$ CI, $0.19-0.90 ; p=0.026)$, and tumor size (HR, 1.83; 95\% CI, 1.29-2.59; $p=0.001)$ were significant factors for one-year DFS.

Table 3. Univariate and multivariate Cox regression analysis for disease-free survival.

\begin{tabular}{|c|c|c|c|c|c|c|c|c|}
\hline \multirow{3}{*}{ Variables } & \multicolumn{4}{|c|}{ One-Year Disease-Free Survival } & \multicolumn{4}{|c|}{ Two-Year Disease-Free Survival } \\
\hline & \multicolumn{2}{|c|}{ Univariate Analysis } & \multicolumn{2}{|c|}{ Multivariate Analysis } & \multicolumn{2}{|c|}{ Univariate Analysis } & \multicolumn{2}{|c|}{ Multivariate Analysis } \\
\hline & HR $(95 \%$ CI $)$ & $p$-Value & HR $(95 \%$ CI) & $p$-Value & HR $(95 \%$ CI) & $p$-Value & HR $(95 \%$ CI) & $p$-Value \\
\hline Age (years) & $0.98(0.96-1.01)$ & 0.223 & & & $0.99(0.96-1.01)$ & 0.295 & & \\
\hline Male sex & $1.34(0.68-2.64)$ & 0.393 & & & $1.14(0.64-2.01)$ & 0.663 & & \\
\hline $\begin{array}{l}\text { Treatment naïve } \\
\text { Cause of HCC }\end{array}$ & $0.35(0.17-0.72)$ & 0.004 & $0.41(0.20-0.85)$ & 0.017 & $0.32(0.17-0.62)$ & 0.001 & $0.37(0.19-0.72)$ & 0.003 \\
\hline Viral vs. Non-viral & $0.90(0.42-1.93)$ & 0.786 & & & $0.81(0.41-1.60)$ & 0.549 & & \\
\hline Cirrhosis & $2.00(0.79-5.06)$ & 0.142 & & & $2.31(0.99-5.36)$ & 0.052 & & \\
\hline Treatment modality & & & & & & & & \\
\hline MWA vs. RFA & $0.47(0.23-0.96)$ & 0.040 & $0.42(0.19-0.90)$ & 0.026 & $0.45(0.23-0.90)$ & 0.024 & $0.41(0.20-0.86)$ & 0.017 \\
\hline CTP class B vs. class A & $1.34(0.48-3.72)$ & 0.581 & & & $1.06(0.38-2.91)$ & 0.916 & & \\
\hline $\operatorname{AFP}(\mathrm{ng} / \mathrm{mL})$ & $1.00(1.00-1.00)$ & 0.019 & $1.00(1.00-1.00)$ & 0.246 & $1.00(1.00-1.00)$ & 0.035 & $1.00(1.00-1.00)$ & 0.280 \\
\hline MELD score & $0.99(0.89-1.11)$ & 0.847 & & & $0.96(0.86-1.08)$ & 0.514 & & \\
\hline Tumor size $(\mathrm{cm})$ & $1.62(1.15-2.28)$ & 0.005 & $1.83(1.29-2.59)$ & 0.001 & $1.53(1.12-2.10)$ & 0.007 & $1.78(1.28-2.47)$ & 0.001 \\
\hline Tumor number & & & & & & & & \\
\hline Multiple vs. single & $1.38(0.73-2.62)$ & 0.321 & & & $1.69(0.98-2.91)$ & 0.057 & & \\
\hline $\begin{array}{l}\text { Perivascular tumor } \\
\text { BCLC stage }\end{array}$ & $1.28(0.68-2.38)$ & 0.446 & & & $1.13(0.64-2.00)$ & 0.672 & & \\
\hline Stage A,B vs. stage 0 & $1.76(0.98-3.16)$ & 0.061 & & & $1.80(1.07-3.01)$ & 0.026 & $1.15(0.57-2.31)$ & 0.694 \\
\hline
\end{tabular}

HR, hazard ratio; CI, confidence interval; HCC, hepatocellular carcinoma; MWA, microwave ablation; RFA radiofrequency ablation; CTP, Child-Turcotte-Pugh; AFP, alpha-fetoprotein; MELD, model for end-stage liver disease; BCLC, Barcelona clinic liver cancer.

Treatment-naïve tumors, treatment modality, AFP level, tumor size, and BCLC stage were associated with two-year DFS in the univariate analysis. As with one-year DFS, treatment-naïve tumors (HR, 0.37; 95\% CI, 0.19-0.72; $p=0.003)$, MWA (HR, $0.41 ; 95 \% \mathrm{CI}$, $0.20-0.86 ; p=0.017)$ and tumor size $(\mathrm{HR}, 1.78,95 \% \mathrm{CI}, 1.28-2.47 ; p=0.001)$ were independent factors for two-year DFS. However, AFP level and BCLC stage had no significant effect on DFS at two years.

\subsection{PSM Analysis}

Finally, we evaluated treatment outcomes according to treatment modalities after PSM. The baseline characteristics, including treatment-naïve tumors and tumor size, were similar between the RFA $(n=42)$ and MWA $(n=42)$ groups after PSM (Table 1$)$. OS at two years was comparable between the two groups (Figure 4A). Meanwhile, DFS at one and two 
years was still significantly better in the MWA group than in the RFA group ( $p=0.018$ and $p=0.023$, respectively) (Figure 4B,C).

(A)

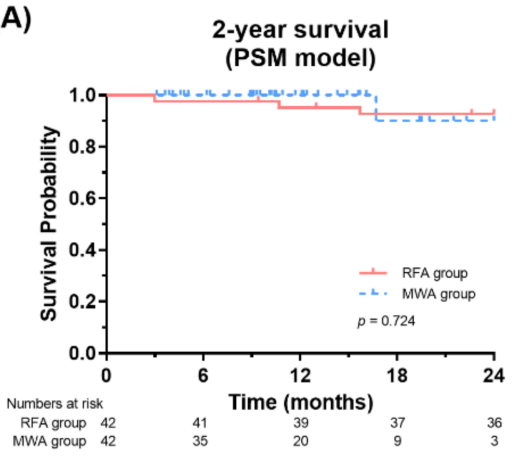

(B)

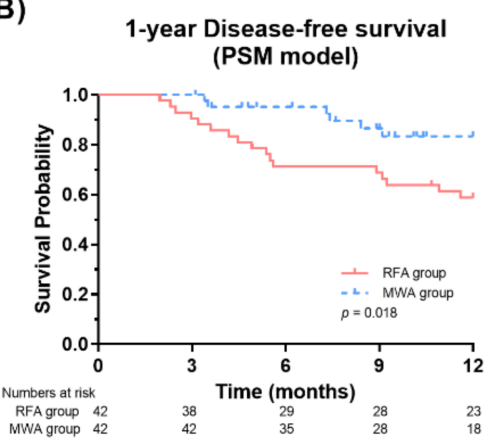

(C)

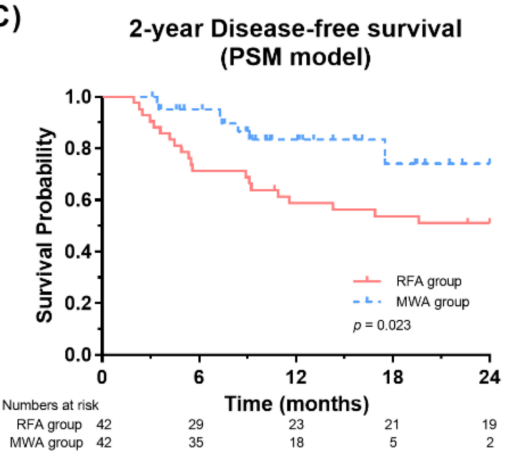

Figure 4. Survival and disease-free survival curves according to treatment modalities in the propensity-score matched cohort $(\mathrm{A}-\mathrm{C})$. RFA, radiofrequency ablation; MWA, microwave ablation.

\section{Discussion}

This is the first study in Korea to compare the outcomes of MWA and RFA therapy for HCC, including patients with recurrent HCC. Our detailed analyses documented better DFS at one and two years with a lower risk of major complications in the MWA group than in the RFA group. Similar trends were observed in subgroups of patients with a high risk of recurrence, perivascular tumor, or small tumor size $(\leq 3 \mathrm{~cm})$. However, the $\mathrm{OS}$ at two years and $\mathrm{CR}$ rate were similar between the two groups. Thus, these findings suggest that MWA could be applied to patients with treatment-naïve and recurrent HCC with an advantage in treating patients with perivascular tumors, small tumors, and a high risk of recurrence.

In our study, MWA was superior to RFA in terms of one- and two-year DFS before and after PSM and was also a favorable factor for DFS at one and two years. The better DFS of MWA might be attributable to the mechanistic advantages of MWA using electromagnetic fields, which causes a rotation of water and polarization of ions that can lead to a larger active heating zone than RFA, and these results were in accordance with previous studies $[12,13,22]$. Moreover, this is the first study to document the superior outcomes of MWA including patients with recurrent HCC. Generally, after the recurrence of HCC, the treatment method is selected following the first-line treatment guidelines [15]. Based on our results, MWA might be also applied to patients with recurrent HCC as an alternative to RFA.

Although MWA could generate a larger ablation zone with better DFS than RFA, MWA resulted in similar two-year OS and CR rates to RFA. As most patients $(n=147,98.0 \%)$ had small tumors within the Milan criteria, OS at two years was more than $90 \%$ in both treatment groups and these high rates of survival might have affected the similar outcomes of both treatments. With longer follow-up, Liu et al. demonstrated that MWA resulted in better five-year OS than RFA in patients within the Milan criteria [22]. Meanwhile, the CR rate of MWA in our study was $94 \%$ and these high CR rates of both treatments without significant differences were in line with previous studies [23].

Interestingly, both groups showed similar rates of complications, although MWA was considered to have a higher rate of complications due to a larger ablation zone. Similar to the findings of previous studies, there were no group differences, and the most common complication in both groups was pain with and without treatment $[17,23,24]$. Moreover, our study demonstrated that the major complication rate in the MWA group was lower than that in the RFA group. This result is different from that of a prior study showing a higher rate of major complications in the MWA group than in the RFA group, which may be due to the fact that the prior study included only patients with treatment-naïve and perivascular tumors [17]. Indeed, our study also included patients with recurrent HCC and our results suggested that MWA was not only effective but also a safe treatment modality 
for patients with treatment-naïve and recurrent HCC. Further studies with large number of patients are necessary to validate our results about the complications of MWA in patients with treatment-naïve and recurrent HCC.

It remains uncertain which patients are more suitable for MWA than RFA. As RFA is considered susceptible to the "heat-sink" effect and is less effective in perivascular tumors, MWA has been evaluated to be more useful in perivascular tumors. In accordance with several studies, our study also showed better DFS in the MWA group than in the RFA group, and MWA might be considered the technique of choice for perivascular tumors [12,17]. In patients with cirrhosis or an increased level of AFP, which are both known risk factors for HCC [25], MWA also demonstrated better DFS than RFA. Taken together, MWA could be applied as an alternative treatment to RFA for patients at high risk for recurrence and with perivascular tumor.

Moreover, MWA could create a larger ablation zone, and the outcomes of MWA related to tumor size have been studied. According to our study, MWA could be an alternative treatment for patients with an HCC tumor size $\leq 3 \mathrm{~cm}$, as supported by previous studies [26]. However, most patients $(n=138,92.0 \%)$ in our study had a small tumor size $(\leq 3 \mathrm{~cm})$; therefore, further studies are needed to determine the most effective tumor size for MWA. Since MWA can reach its targeted temperature faster, MWA may be beneficial for treating multiple HCCs. However, MWA showed marginally better DFS than RFA in patients with BCLC stage $\mathrm{A}$ and $\mathrm{B}$, and including patients with recurrent $\mathrm{HCC}$ might contributed to these results due to the increased the possibility of recurrence [7].

Our study has several limitations. First, this study had a retrospective design. This study also had a small number of included patients and a short follow-up duration. Considering small tumor size and early stage of included patients who underwent RFA or MWA, two-year OS might not enough to determine survival differences between RFA and MWA. However, this is the first study to evaluate the efficacy and safety of MWA compared to RFA in Korea. Moreover, for the first time, we included patients with treatment-naïve and recurrent HCC to document the real-life experience of MWA. With detailed analyses including PSM, our study can provide practical information about the outcomes of MWA compared to RFA.

In conclusion, MWA therapy for treatment-naïve and recurrent HCC is safe and provides a better DFS than RFA. Moreover, in patients with a high risk of recurrence, perivascular tumors, or small tumor size, MWA might be a technique of choice and an alternative to RFA with a lower rate of early recurrence. Further large-scale studies are necessary to determine the optimal patient groups for MWA.

Supplementary Materials: The following supporting information can be downloaded at: https: / / www.mdpi.com/article/10.3390/jcm11020302/s1. Figure S1: Study flow chart; Figure S2: The comparison of recurrent site between the MWA and RFA group among patients with recurrence; Table S1: Treatment modalities after recurrence in recurrent patients of the RFA and MWA groups for two years follow-up.

Author Contributions: Study concept and design: S.H.C.; acquisition of data: S.K.L., D.J.C.; analysis and interpretation of data: S.K.L., D.J.C. and S.H.C.; drafting of the manuscript: S.K.L.; study supervision: S.H.C. All authors have read and agreed to the published version of the manuscript.

Funding: This study was supported by Basic Science Research Program through the National Research Foundation of Korea (NRF) funded by the Ministry of Education (No. 2021R1I1A1A01050954).

Institutional Review Board Statement: This study was approved by the Institutional Review Board of Yeouido St. Mary's Hospital (SC21RISI0023).

Informed Consent Statement: Patient consent was waived due to retrospective study design.

Data Availability Statement: Data are not available due to ethical issues.

Conflicts of Interest: The authors declare no conflict of interest. 


\section{References}

1. Jung, K.W.; Won, Y.J.; Kong, H.J.; Lee, E.S. Prediction of Cancer Incidence and Mortality in Korea, 2019. Cancer Res. Treat 2019, 51, 431-437. [CrossRef] [PubMed]

2. Siegel, R.L.; Miller, K.D.; Jemal, A. Cancer statistics, 2020. CA Cancer J. Clin. 2020, 70, 7-30. [CrossRef] [PubMed]

3. De Angelis, R.; Sant, M.; Coleman, M.P.; Francisci, S.; Baili, P.; Pierannunzio, D.; Trama, A.; Visser, O.; Brenner, H.; Ardanaz, E.; et al. Cancer survival in Europe 1999-2007 by country and age: Results of EUROCARE-5—A population-based study. Lancet Oncol. 2014, 15, 23-34. [CrossRef]

4. Kim, T.H.; Kim, S.Y.; Tang, A.; Lee, J.M. Comparison of international guidelines for noninvasive diagnosis of hepatocellular carcinoma: 2018 update. Clin. Mol. Hepatol. 2019, 25, 245-263. [CrossRef]

5. Haq, M.I.; Drake, T.M.; Goh, T.L.; Ahmed, A.; Forrest, E.; Barclay, S.; Gillespie, R.; Priest, M.; Evans, J.; Graham, J.; et al. Effect of Hepatocellular Carcinoma Surveillance Programmes on Overall Survival in a Mixed Cirrhotic UK Population: A Prospective, Longitudinal Cohort Study. J. Clin. Med. 2021, 10, 2770. [CrossRef] [PubMed]

6. Mazzaferro, V.; Lencioni, R.; Majno, P. Early hepatocellular carcinoma on the procrustean bed of ablation, resection, and transplantation. Semin. Liver Dis. 2014, 34, 415-426.

7. Kim, J.; Kang, W.; Sinn, D.H.; Gwak, G.Y.; Paik, Y.H.; Choi, M.S.; Lee, J.H.; Koh, K.C.; Paik, S.W. Substantial risk of recurrence even after 5 recurrence-free years in early-stage hepatocellular carcinoma patients. Clin. Mol. Hepatol. 2020, 26, 516-528. [CrossRef]

8. Pompili, M.; Saviano, A.; de Matthaeis, N.; Cucchetti, A.; Ardito, F.; Federico, B.; Brunello, F.; Pinna, A.D.; Giorgio, A.; Giulini, S.M.; et al. Long-term effectiveness of resection and radiofrequency ablation for single hepatocellular carcinoma $\leq 3 \mathrm{~cm}$. Results of a multicenter Italian survey. J. Hepatol. 2013, 59, 89-97. [CrossRef]

9. Seror, O. Ablative therapies: Advantages and disadvantages of radiofrequency, cryotherapy, microwave and electroporation methods, or how to choose the right method for an individual patient? Diagn Interv. Imaging 2015, 96, 617-624. [CrossRef] [PubMed]

10. Kang, T.W.; Lim, H.K.; Lee, M.W.; Kim, Y.S.; Rhim, H.; Lee, W.J.; Gwak, G.Y.; Paik, Y.H.; Lim, H.Y.; Kim, M.J. Aggressive Intrasegmental Recurrence of Hepatocellular Carcinoma after Radiofrequency Ablation: Risk Factors and Clinical Significance. Radiology 2015, 276, 274-285. [CrossRef]

11. Crocetti, L.; de Baere, T.; Lencioni, R. Quality improvement guidelines for radiofrequency ablation of liver tumours. Cardiovasc. Interv. Radiol. 2010, 33, 11-17. [CrossRef] [PubMed]

12. Izzo, F.; Granata, V.; Grassi, R.; Fusco, R.; Palaia, R.; Delrio, P.; Carrafiello, G.; Azoulay, D.; Petrillo, A.; Curley, S.A. Radiofrequency Ablation and Microwave Ablation in Liver Tumors: An Update. Oncologist 2019, 24, e990-e1005. [CrossRef] [PubMed]

13. Habibollahi, P.; Sheth, R.A.; Cressman, E.N.K. Histological Correlation for Radiofrequency and Microwave Ablation in the Local Control of Hepatocellular Carcinoma (HCC) before Liver Transplantation: A Comprehensive Review. Cancers 2020, 13, 104. [CrossRef]

14. Marrero, J.A.; Kulik, L.M.; Sirlin, C.B.; Zhu, A.X.; Finn, R.S.; Abecassis, M.M.; Roberts, L.R.; Heimbach, J.K. Diagnosis, Staging, and Management of Hepatocellular Carcinoma: 2018 Practice Guidance by the American Association for the Study of Liver Diseases. Hepatology 2018, 68, 723-750. [CrossRef] [PubMed]

15. EASL Clinical Practice Guidelines: Management of hepatocellular carcinoma. J. Hepatol. 2018, 69, 182-236. [CrossRef]

16. Lee, S.; Kang, T.W.; Cha, D.I.; Song, K.D.; Lee, M.W.; Rhim, H.; Lim, H.K.; Sinn, D.H.; Kim, J.M.; Kim, K. Radiofrequency ablation vs. surgery for perivascular hepatocellular carcinoma: Propensity score analyses of long-term outcomes. J. Hepatol. 2018, 69, 70-78. [CrossRef]

17. Feng, Y.; Wang, L.; Lv, H.; Shi, T.; Xu, C.; Zheng, H.; Qi, J.; Zhao, X.; Li, J.; Gao, Y.; et al. Microwave ablation versus radiofrequency ablation for perivascular hepatocellular carcinoma: A propensity score analysis. HPB 2021, 23, 512-519. [CrossRef]

18. 2018 Korean Liver Cancer Association-National Cancer Center Korea Practice Guidelines for the Management of Hepatocellular Carcinoma. Gut Liver 2019, 13, 227-299. [CrossRef]

19. Lencioni, R.; Llovet, J.M. Modified RECIST (mRECIST) assessment for hepatocellular carcinoma. Semin. Liver Dis. 2010, 30, 52-60. [CrossRef]

20. Rossi, S.; Ravetta, V.; Rosa, L.; Ghittoni, G.; Viera, F.T.; Garbagnati, F.; Silini, E.M.; Dionigi, P.; Calliada, F.; Quaretti, P.; et al. Repeated radiofrequency ablation for management of patients with cirrhosis with small hepatocellular carcinomas: A long-term cohort study. Hepatology 2011, 53, 136-147. [CrossRef]

21. Sacks, D.; McClenny, T.E.; Cardella, J.F.; Lewis, C.A. Society of Interventional Radiology clinical practice guidelines. J. Vasc Interv. Radiol. 2003, 14, S199-S202. [CrossRef] [PubMed]

22. Liu, W.; Zheng, Y.; He, W.; Zou, R.; Qiu, J.; Shen, J.; Yang, Z.; Zhang, Y.; Wang, C.; Wang, Y.; et al. Microwave vs radiofrequency ablation for hepatocellular carcinoma within the Milan criteria: A propensity score analysis. Aliment. Pharmacol. Ther. 2018, 48, 671-681. [CrossRef] [PubMed]

23. Facciorusso, A.; Abd El Aziz, M.A.; Tartaglia, N.; Ramai, D.; Mohan, B.P.; Cotsoglou, C.; Pusceddu, S.; Giacomelli, L.; Ambrosi, A.; Sacco, R. Microwave Ablation Versus Radiofrequency Ablation for Treatment of Hepatocellular Carcinoma: A Meta-Analysis of Randomized Controlled Trials. Cancers 2020, 12, 3796. [CrossRef] [PubMed]

24. Liang, P.; Wang, Y.; Yu, X.; Dong, B. Malignant liver tumors: Treatment with percutaneous microwave ablation-Complications among cohort of 1136 patients. Radiology 2009, 251, 933-940. [CrossRef] 
25. Fujiwara, N.; Friedman, S.L.; Goossens, N.; Hoshida, Y. Risk factors and prevention of hepatocellular carcinoma in the era of precision medicine. J. Hepatol. 2018, 68, 526-549. [CrossRef]

26. Nault, J.C.; Sutter, O.; Nahon, P.; Ganne-Carrié, N.; Séror, O. Percutaneous treatment of hepatocellular carcinoma: State of the art and innovations. J. Hepatol. 2018, 68, 783-797. [CrossRef] 